\title{
Environmentally friendly and roll-processed flexible organic solar cells based on PM6:Y6.
}

\author{
Marcial Fernández Castro, Jean Truer, Moisés Espíndola Rodríguez, and Jens Wenzel Andreasen. \\ Technical University of Denmark, Department of Energy Conversion and Storage \\ Organic Solar Cells (OSCs) have reached the highest efficiencies using lab-scale on active areas far below $0.1 \mathrm{~cm} 2$. This tends \\ to widen the so-called "lab-to-fab gap", which is one of the most important challenges to make OSCs competitive. The most \\ commonly used fabrication technique is spin-coating, which has poor compatibility with large-scale techniques and \\ substantial material waste. Moreover, other techniques such as blade or slot-die coating are much more suitable for roll-to- \\ roll manufacturing processes, which is one of the advantages the technology has compared, for example, to silicon solar \\ cells. However, only a few studies report solar cells using these fabrication techniques. Additionally, for the environmentally \\ friendly OSC scale-up, inks based on non-hazardous solvent systems are needed. In this work, slot-die coating has been \\ chosen to coat the PM6:Y6 active layer, using o-xylene, a green solvent, without additives. The optimal coating parameters \\ are defined through fine-tuning of the coating parameters, such as the drying temperature and solution concentration. \\ Moreover, ternary devices with PCBM, and fully printed devices are also fabricated. Power conversion efficiencies of $6.26 \%$ \\ and 7.16\% are achieved for binary PM6:Y6 and ternary PM6:Y6:PCBM devices, respectively.
}

Keywords: organic solar cells, green solvent, additive-free, roll-process, flexible, large area, PM6, Y6

\section{Introduction}

Higher efficiencies are being reached in Organic Solar Cells (OSCs) every semester especially due to the non-fullerene acceptors, which allow fine-tuning of their energy levels and light absorption with different donor materials ${ }^{1-4}$. Moreover, NFAs can reduce the $V_{O C}$ losses and present a stronger and wider visible-NIR absorption 5,6 .

OSCs also present interesting properties compared to other photovoltaic (PV) technologies, such as their low cost, their mechanical flexibility (making them roll-to-roll compatible), lightweight, the possibility of being semi-transparent, and their very short Energy Payback Time (EPBT). The EPBT is the time needed to give back the energy used for manufacturing the device and can reach the order of weeks for organic photovoltaics ${ }^{7-11}$. This short EPBT is related to the small amount of energy and material needed for the manufacture.

All of this makes OSCS one the most promising PV technologies, driving a lot of research attention in order to achieve their industrialization. However, a large efficiency gap is still to be overcome between solar cells produced with lab-scale techniques and the ones produced using industrial-compatible processes. ${ }^{12-14}$ The most reliable scalable fabrication techniques for OSCs are slotdie coating, blade-coating, or even spray and aerosol coating, due to their compatibility with roll-to-roll manufacturing. However, slot-die coating is preferred, as it is possible to coat with a one-dimensional pattern in the form of one or more stripes with a well-defined width and with almost no material waste. ${ }^{12,15-17}$. Therefore, in order to contribute to minimizing the "lab-to-fab gap", slot-die coating should be the choice for the highest efficiency systems.

A lot of new donors and acceptors appeared recently on the market, including PM6 donor and Y6 acceptor materials (chemical structures shown in Figure S1). They showed excellent efficiencies over $15 \%$ on rigid substrates when using green solvents as o-xylene (O-XY), and without additives, using an inverted structure of glass/ITO/ZnO/PM6:Y6/ $/ \mathrm{MoO}_{3} / \mathrm{Al}{ }^{18}$. Other device structures were used with different transport layers and electrodes and efficiencies between 10 and $16 \%$ were achieved ${ }^{1}$. PM 6 absorption matches well the highest spectral irradiance point of the solar spectrum, while Y6 complements this absorption in the red part of the spectrum (Figure 1(right). Therefore, PM6:Y6 material combination is a firm candidate to minimize the gap between efficiencies obtained using different manufacturing processes ${ }^{1,18-20}$. To the best of our knowledge, there is still a need to transfer PM6:Y6 devices to roll-processing on flexible substrates, using scalable fabrication techniques as slot-die coating. However, the PM6 exhibits temperature-dependent aggregation (TDA) behavior and therefore needs to be heated to remain liquid during all the slot-die coating procedures ${ }^{18}$. In this case, the substrate, syringe, tube, and the slot-die head are heated during the coating following the temperature-controlled slot-die coating previously reported by our group ${ }^{17}$.

In this work, we manufacture OSCs using large-scale compatible processes on flexible lightweight substrates. Intending to achieve high efficiencies using scalable techniques for the manufacture of 

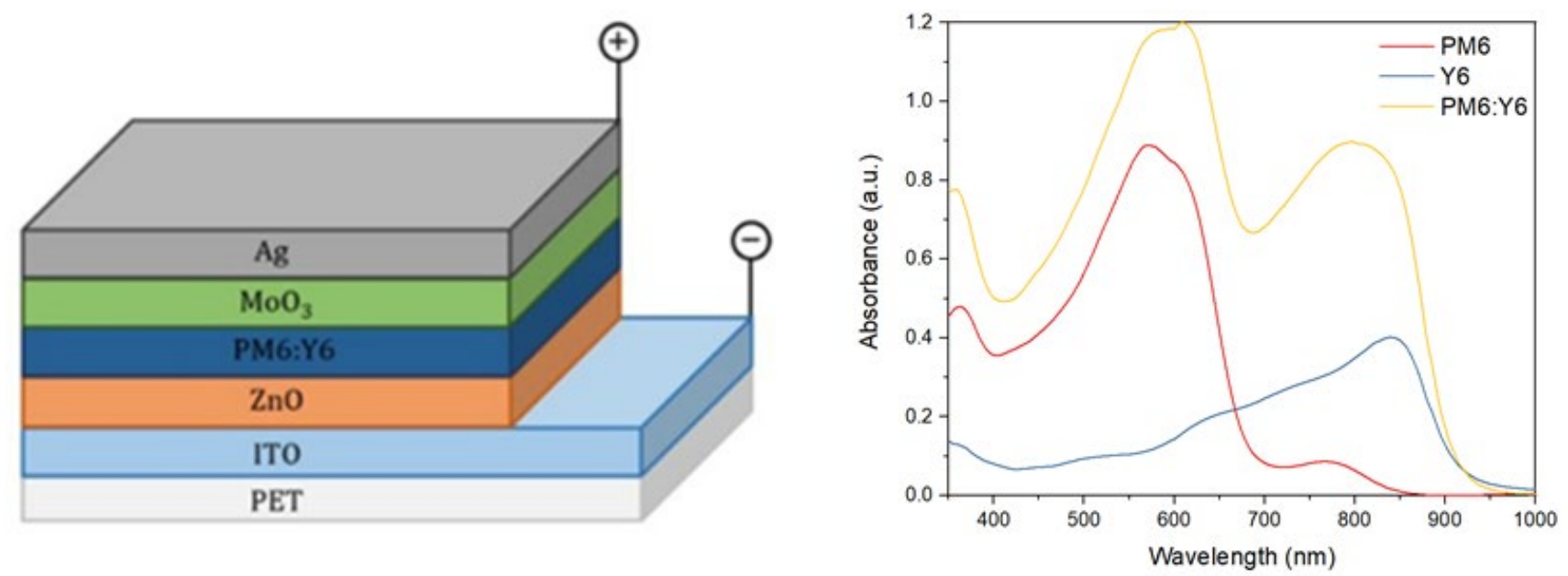

Figure 1: (left) Representation of the inverted OSC used for this study. (right) Absorbance of photoactive materials in O-XY (16 mg/mL) slot-die coated at $100{ }^{\circ} \mathrm{C}$.

OSCs, we hope to contribute to closing the "lab-to-fab gap" and finally bring this technology to the edge of commercialization. ${ }^{12,14}$ For the first time, slot-die coated flexible devices based on PM6:Y6 were fabricated in open-air, with efficiencies up to $7 \%$ when paired with fullerene acceptors in a ternary device. After more than $1000 \mathrm{~h}$ of storage, the devices suffer almost no degradation, keeping over $99 \%$ of the initial efficiency.

\section{Experimental}

\section{Materials}

The materials involved in this study were purchased and used directly without purification. The active layer (AL) materials, PM6 (batch YY19260CH100) and Y6 (batch DW7038S) were purchased from 1Material Inc. whereas $\mathrm{PB}_{61} \mathrm{BM}$ (batch $\mathrm{M} 111$ ) was purchased from Ossila. The materials used for the transport layers are $\mathrm{ZnO}$ nanoparticle ink (from Sigma-Aldrich) and $\mathrm{MoO}_{3}$ pellets (from Kurt J. Lesker Co.). The flexible substrate is made of pre-patterned ITOcoated polyethylene terephthalate (PET), purchased from FOM Technologies. Silver for evaporation of the back electrode is pure silver bought from Sigma-Aldrich. Silver paste for flexographic printing is a heat-curing paste (DuPont 5025). The solvent used in this study to prepare the AL solution (ink) was O-XY (with a $\geq 98 \%$ purity) and was purchased from Sigma-Aldrich.

\section{Solar Cell fabrication}

As shown in Figure 1(left), the device is made with the following inverted geometry: ITO/ZnO/PM6: $\mathrm{Y} 6 / \mathrm{MoO}_{3} / \mathrm{Ag}$. The $\mathrm{ZnO}$ Electron Transport Layer (ETL) and AL are coated using a homemade rollcoater fabricated with the objective of coating at fully compatible roll-to-roll conditions at a laboratory scale.

A syringe pump is used to control the flow rate of the inks. The full setup is made in order to be able to control the speed and heat of the drum and the drum-to-head distance. Since the ink needs to remain hot, a hot tube is necessary to bring the AL from the syringe to the slot-die head.

The $\mathrm{ZnO}$ nanoparticle ink (ETL) was coated at a substrate temperature of $75^{\circ} \mathrm{C}$ with a $0.06 \mathrm{~mL} / \mathrm{min}$ flow rate and a drum speed of $1.08 \mathrm{~m} / \mathrm{min}$. It is then annealed in an oven at $100{ }^{\circ} \mathrm{C}$ for $10 \mathrm{~min}$ in air. The AL PM6:Y6 (ratio 1:1) was coated at different temperatures $\left(60,80,100,120^{\circ} \mathrm{C}\right)$ and concentrations $(12,16,20,24,28 \mathrm{mg} / \mathrm{mL})$ for the study. The head, syringe, drum, and tube were kept at the same temperature, for each temperature under investigation as in previous studies it was demonstrated this leads to an optimal performance ${ }^{17,21}$. The drum speed was kept at $1.5 \mathrm{~m} / \mathrm{min}$ and the solution was coated with a $0.12 \mathrm{~mL} / \mathrm{min}$ flow rate. Finally, $10 \mathrm{~nm}$ of $\mathrm{MoO}_{3}$ as Hole Transport Layer (HTL) and $150 \mathrm{~nm}$ of Ag were thermally evaporated under vacuum at a base pressure of $2 \times 10^{-5} \mathrm{mbar}$, through a shadow mask. The materials were sequentially evaporated from different tungsten boats, an INFICON XTM/2 system was used to monitor the deposition rate and thickness.

Before placing the devices in the evaporator, different parts of the one-meter-long AL are selected to fabricate solar cells. Up to 24 solar cells are fabricated per evaporation. At least 8 devices are then encapsulated. The devices have an approximate active area of 0.8 $\mathrm{cm}^{2}$.

\section{Activation and encapsulation}

The cells are characterized directly after the evaporation. However, before encapsulation, a last step is necessary, an activation process that consists of a reverse bias current through the device ( $-5 \mathrm{~V}$ with a maximum current of $100 \mathrm{~mA}$ ). This ensures the stabilization of the $\mathrm{J}-\mathrm{V}$ curve before the encapsulation step. Encapsulation is made using epoxy-based glue, EPXR, made of bisphenol A diglycidyl ether and Chissonox 221 monomers. Two glass slides are used to protect both the up and down sides of the device where the EPOXY is applied, enabling the formation of an oxygen and humidity barrier once cured. Curing is made under UV light for approximately $5 \mathrm{~min}$, with a temperature that reaches $66 \pm 0.5^{\circ} \mathrm{C}$ during the process. ${ }^{17}$

\section{Characterization}

The current density-voltage (J-V) curves were measured with a Keithley 2400 source meter ( $50 \mathrm{mV}$ step reverse to forward) and a solar simulator, calibrated to $1000 \mathrm{~W} / \mathrm{m}^{2}$ with a reference cell (monocrystalline silicon certified by Fraunhofer) under AM1.5G illumination. To ensure that the area is accurate and kept the same 
between different coatings, a shadow mask purchased from Ossila (E2002A1) was used to define an aperture area of $0.3996 \mathrm{~cm}^{2}$ Averages include statistics of eight devices.

The External Quantum Efficiency (QE) and absorbance measurements are done with a QEX10 system (PV Measurements Inc.) from 300 to $1100 \mathrm{~nm}$ (5 nm step size) calibrated with a Si photodiode.

For the light intensity measurements, we increased the intensity of the solar simulator lamp from $62.14 \mathrm{~mW} / \mathrm{cm}^{2}$ to $147.33 \mathrm{~mW} / \mathrm{cm}^{2}$, measuring $\mathrm{J}-\mathrm{V}$ characteristics at different intermediate points.

The thickness measurements are done with a DS95-50 AFM instrument from the Danish Micro Engineering (DME). The dimension of the scans is a $5 \times 5 \mu \mathrm{m}^{2}$ surface while using the noncontact mode.

\section{Results and discussion}

We demonstrate the feasibility of slot-die coating PM6:Y6 in open air on flexible, using a green solvent without additives. The optimization of the coating parameters in a roll-platform is presented. Our results can be easily transferred to a roll-to-roll platform which is key to increasing the competitiveness of this technology, now that high efficiencies were reached using lab-scale techniques 10,12,17,22.

First, we present the coating temperature optimization, then with the optimum temperature we investigate the effect of the $A L$ thickness on the performance of the devices. In the end, we demonstrate the advantages of introducing a small amount of a fullerene acceptor to the blend also coated in open air on flexible substrates. The advantages unfold an improved PCE and increased stability.

\section{Active layer optimization}

It has been previously shown that efficiencies over $15 \%$ can be achieved using PM6:Y6 as active materials using hot slot-die coating on ITO-coated glass substrate at the same temperature as the solution. These efficiencies were achieved with a concentration of 16 $\mathrm{mg} / \mathrm{mL}$ in the green solvents $\mathrm{O}-\mathrm{XY}$, or trimethylbenzene (TMB) without the use of additives ${ }^{1,18}$. In this study, $\mathrm{O}-\mathrm{XY}$ green solvent was selected as our target solvent and $16 \mathrm{mg} / \mathrm{ml}$ solutions were prepared as a starting point. The D/A ratio was fixed at 1:1, as the highest reported efficiencies for the system were achieved with that ratio. $1,18-20,23$

Concerning the coating parameters of the $\mathrm{AL}$, we fixed the solution flow rate and the drum speed, in values that ensured a homogenous layer and no aggregation during the coating. We screen the $A L$ coating temperature (slot-die head temperature) from $60^{\circ} \mathrm{C}$ to 120 ${ }^{\circ} \mathrm{C}$ matching it with the drum and tube temperature. The coating temperature plays an important role in the drying kinetics of the solvent, directly affecting the morphology of the $\mathrm{AL}$, and so, the donor and acceptor packing. This strongly influences the absorbance of the $\mathrm{AL}$ and consequently the performance of the final devices, as morphology and efficiency are closely related. ${ }^{24-30}$ Figure S2 shows absorption measurements of dried blend films slot-die coated at different temperatures. The dried blends exhibit strong absorption in the $460 \mathrm{~nm}$ to $870 \mathrm{~nm}$. The PM6 absorption is in the $\sim 460-650$ $\mathrm{nm}$ region and the $\mathrm{Y} 6$ absorption in the $\sim 660-870 \mathrm{~nm}$ region (Figure 1b). No blue or redshift is observed in the spectra of the dried films coated with different temperatures. The absorption of PM6 in the dried films is the same for the different coating temperatures as shown in the normalized spectra in Figure S2b. In contrast with the absorption enhancement of the spectral region of $\mathrm{Y} 6$ absorption with $100{ }^{\circ} \mathrm{C}$ and $120^{\circ} \mathrm{C}$ coating temperatures.

$\mathrm{J}-\mathrm{V}$ curves of representative inverted configuration devices with $\mathrm{AL}$ coated at different temperatures and their external quantum efficiency measurements (EQE) are shown in Figure 2. It is worth mentioning that annealing $\left(120^{\circ} \mathrm{C}, 3\right.$ minutes) of the encapsulated devices improves the $\mathrm{J}_{\mathrm{SC}}$ of the devices with $\mathrm{AL}$ coated $60^{\circ} \mathrm{C}$ and 80 ${ }^{\circ} \mathrm{C}$ (Figure S3). On the other hand, the devices fabricated at $100{ }^{\circ} \mathrm{C}$ and $120^{\circ} \mathrm{C}$ do not improve after annealing, as can be seen in Figure S4. Thermal annealing is usually correlated with enhanced polymer
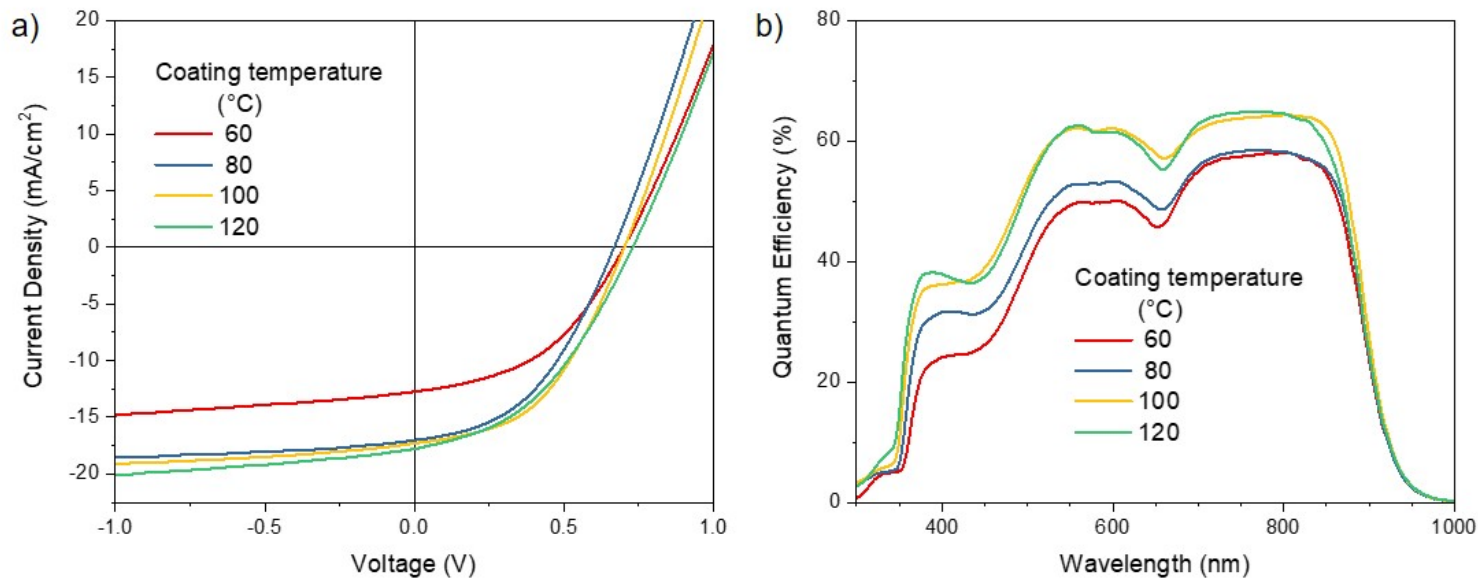

Figure 2: a) J-V curve comparison between devices coated at different temperatures. Measurements done under simulated solar light AM1.5G and $1000 \mathrm{~W} / \mathrm{m} 2$ and b) External Quantum Efficiency (QE) of PM6:Y6 solar cells coated at different temperatures 
a)

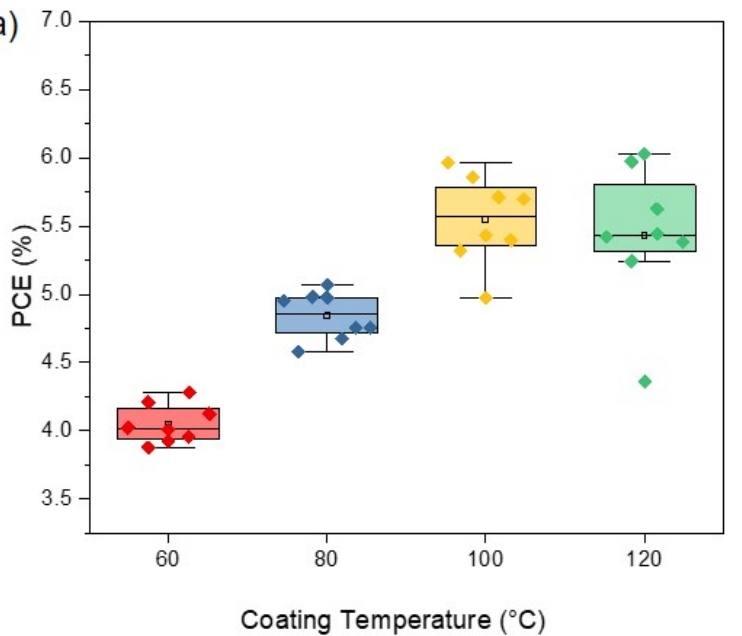

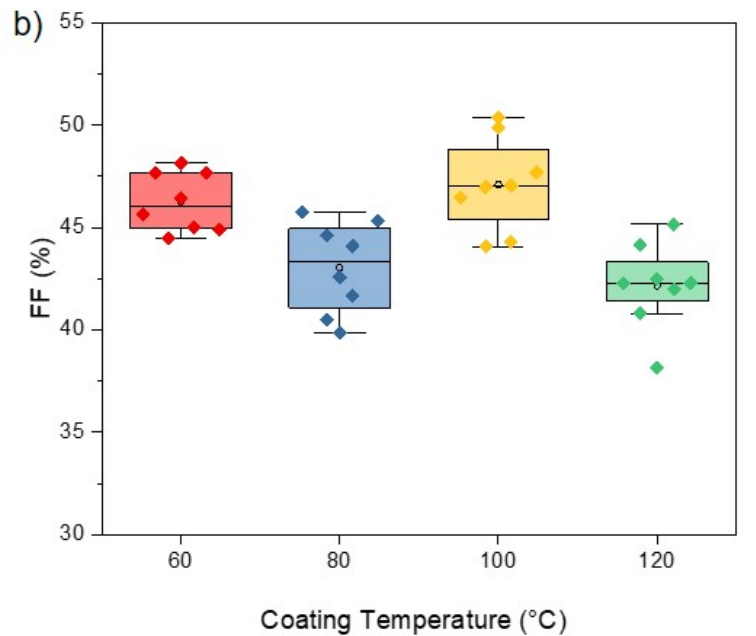

Figure 3: Statistic representation of the measured PV parameters of PM6:Y6 devices fabricated at different temperatures: a) Power Conversion Efficiency (PCE) and b) Fill Factor (FF)

crystallinity ${ }^{30}$, suggesting that when coating at low temperatures, an extra annealing step is necessary, whereas coating at a higher temperature, might impact favorably the molecular packing and texture of the AL during drying ${ }^{18,31}$.
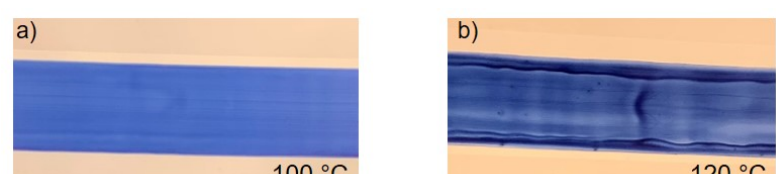

$100^{\circ} \mathrm{C}$

$120^{\circ} \mathrm{C}$

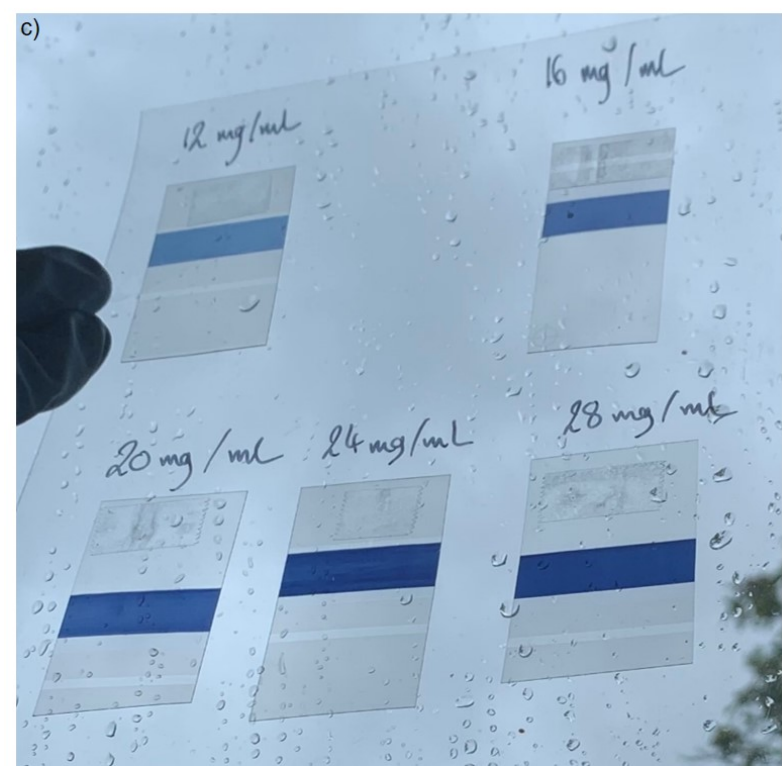

Figure 4: PM6:Y6 slot die coated at a) $100{ }^{\circ} \mathrm{C}$ and b) $120^{\circ} \mathrm{C}$. c) Effect of the AL thickness on the transparency of the ITO/ZnO/AL stack against a window light.

As can be noticed in Figure 2(b), the EQE of the final devices increases when coating at higher temperatures, indicating that coating the $\mathrm{AL}$ at higher temperatures induces better carrier collection, thus a higher current. The short-short circuit current is higher at higher coating temperatures (Table 1). However, the average FF value of $42.16 \%$ is lower for the $120{ }^{\circ} \mathrm{C}$ coating than for the $100{ }^{\circ} \mathrm{C}$ coating
(47.10\%). This makes the average PCE of our devices at $100^{\circ} \mathrm{C}$ slightly higher than the obtained when coating at $120^{\circ} \mathrm{C}$. In order to better visualize the tendency of the performance with temperature variations, box plots with statistical data of the PCE and FF evolution of eight devices are shown in Figure 3. Each box indicates the first to the third quartile, whereas the median is indicated as a horizontal line and the average as a square. The PCE shows a clear improvement trend towards higher coating temperature that saturates around 100 ${ }^{\circ} \mathrm{C}$ while the $\mathrm{FF}$ remains around $45 \%$ with the lowest average value of $42.16 \%$ for the devices coated at $120{ }^{\circ} \mathrm{C}$. That can be explained by taking a detailed look at the dried active layer stripe (Figure $4 a, b$ ). When increasing the temperature above $100^{\circ} \mathrm{C}$, the solvent is drying much faster, inducing the aggregation of the materials on both edges of the $\mathrm{AL}$, perpendicular to the coating direction. This effect creates inhomogeneities along the stripe, which directly impacts the fill factor of the final devices. Therefore, based on the higher PCE, a homogeneous layer, and the lack of the extra step of thermal annealing, the optimal temperature was fixed at $100{ }^{\circ} \mathrm{C}$.

Table 1: PV parameters extracted from the J-V curves of PM6:Y6 devices fabricated at different temperatures. Average values are calculated from 8 devices, and the standard deviation is shown as error.

\begin{tabular}{|c|c|c|c|c|}
$\begin{array}{c}\text { Substrate } \\
\text { temperature }\left({ }^{\circ} \mathrm{C}\right)\end{array}$ & 60 & 80 & 100 & 120 \\
\hline $\mathrm{PCE}(\%)$ & $4.05 \pm$ & $4.84 \pm$ & $5.54 \pm$ & $5.43 \pm$ \\
& 0.13 & 0.16 & 0.30 & 0.48 \\
\hline $\mathrm{V}_{\text {oc }}(\mathrm{V})$ & $0.69 \pm$ & $0.71 \pm$ & $0.71 \pm$ & $0.72 \pm$ \\
& 0.01 & 0.02 & 0.01 & 0.01 \\
\hline $\mathrm{J}_{\mathrm{SC}}\left(\mathrm{mA} / \mathrm{cm}^{2}\right)$ & $12.67 \pm$ & $16.51 \pm$ & $16.66 \pm$ & $17.79 \pm$ \\
\hline $\mathrm{J}_{\mathrm{Sc}} \mathrm{EQE}^{2}\left(\mathrm{~mA} / \mathrm{cm}^{2}\right)$ & 0.34 & 0.94 & 0.65 & 0.85 \\
\hline $\mathrm{FF}(\%)$ & 46.82 & 16.69 & 19.17 & 18.96 \\
\hline $\mathrm{PCE}_{\max }(\%)$ & 1.35 & $43.05 \pm$ & $47.10 \pm$ & $42.16 \pm$ \\
\hline & 4.28 & 5.09 & 2.13 & 1.97 \\
\hline
\end{tabular}


Charge carrier recombination plays a crucial role in the final PV parameters, as recombination means the charges are being lost before being collected at the electrodes. The degree and dominant type of recombination can be studied through light intensity dependence of the current density and open-circuit voltage 32 .

In Figure $\mathrm{S6a}$, the bimolecular recombination is investigated using the following relation $\mathrm{J}_{\mathrm{Sc}} \propto \mathrm{P}_{\text {in }}{ }^{\mathrm{S}}$ where $\mathrm{P}_{\text {in }}$ is the incident light intensity. The exponential factor $S$ will be equal to the unit for an ideal device, stating that all the charges generated are collected by the corresponding electrodes. The further away $\mathrm{S}$ gets from one, the more dominant is the bimolecular recombination in the blend. When comparing the devices fabricated through the temperature optimization, the $S$ value closest to the unit $(S=0.978$ ) is obtained coating at $100{ }^{\circ} \mathrm{C}$, implying that one of the reasons for the optimized performance is due to reduced bimolecular recombination.

In order to obtain independent and complementary information of the recombination processes taking place in our OSCS coated at different temperatures, we estimate the recombination mechanism at open-circuit voltage. At such condition (zero current) all photocarriers recombine, the type of recombination can be extracted from the slope of the plot $V_{\text {oc }}$ versus $\ln \left(P_{\text {in }}\right)^{32}$.

The slope is obtained using a linear fitting, the closer the slope is to $\mathrm{kT} / \mathrm{q}$, second-order recombination is the most likely mechanism. If the slope $>k T / q$ the most likely trap-assisted recombination is. Where $k, T$, and $q$ are the Boltzmann constant, the absolute temperature, and the element charge respectively. These suggest that the trap-assisted recombination in our devices is the dominant one (Figure S6b), as all the obtained slope values are higher than $\mathrm{kT} / \mathrm{q}$. Regrettably, there is not a clear tendency in the $s$ values. Therefore, based on the higher PCE, the ease of coating a homogeneous layer, and the lack of the extra thermal annealing steps, the optimal temperature was fixed at $100^{\circ} \mathrm{C}$.

The AL thickness also plays an important role in device efficiency. A thick AL is responsible for increased light absorption, and it will also make the final device more robust, which is important when roll-toroll is used, like pressure, bending, and stretching occurs during the process. However, thinner AL layers allow to have semi-transparent devices which are interesting for many applications ${ }^{7-9,33-35}$, and also reduce the charge carriers' pathways, decreasing the probability of recombination before being collected in the electrodes. In this study, AL layers with four different thicknesses are investigated.

As it is shown in Figure 4c, we chose a wide range of concentrations leading to semi-transparent thin layers to thicker and more opaque layers. The absorption peaks of both materials can be clearly distinguished in Figure 57, being the maximum absorption of the donor at $\sim 625 \mathrm{~nm}$ and the maximum of the acceptor at around 820 $\mathrm{nm}$. The respective J-V curves for representative devices are shown in Figure S7b, whereas a summary of PV parameters of PM6:Y6 based solar cells with different AL thicknesses are shown in Table 2 .
Table 2: PV parameters for PM6:Y6-based OSCs devices prepared with different AL thicknesses. The averages are obtained from 8 devices.

\begin{tabular}{c|c|c|c|c|c|}
$\begin{array}{c}\text { AL } \\
\text { thickness } \\
(\mathrm{nm})\end{array}$ & 101 & 116 & 154 & 217 & 323 \\
\hline $\mathrm{PCE}(\%)$ & $4.77 \pm$ & $5.54 \pm$ & $5.70 \pm$ & $5.82 \pm$ & $5.47 \pm$ \\
& 0.41 & 0.30 & 0.18 & 0.25 & 0.36 \\
\hline $\mathrm{V}_{\text {oc }}(\mathrm{V})$ & $0.71 \pm$ & $0.71 \pm$ & $0.72 \pm$ & $0.72 \pm$ & $0.71 \pm$ \\
\hline $\mathrm{J}_{\text {sc }}$ & 0.01 & 0.01 & 0.01 & 0.01 & 0.01 \\
\hline$\left(\mathrm{mA} / \mathrm{cm}^{2}\right)$ & \pm 0.80 & \pm 0.65 & 0.48 & \pm 0.84 & \pm 0.95 \\
\hline $\mathrm{FF}(\%)$ & 41.97 & 47.10 & $45.55 \pm$ & 47.28 & 46.39 \\
\hline $\mathrm{PCE} \mathrm{Emax}_{\max }(\%)$ & 5.44 & 5.96 & 6.11 & 6.26 & 5.81 \\
\hline
\end{tabular}

The box plots of the PV parameters are shown in Figure 5. As the thickness of the AL increases from $101 \mathrm{~nm}$ to $217 \mathrm{~nm}$, the average PCE also increases. This is directly related to the increase in the average short circuit current density (Figure $5 c$ ) which reaches its maximum at $154 \mathrm{~nm}\left(17.30 \mathrm{~mA} / \mathrm{cm}^{2}\right)$ of AL thickness, to decrease again slightly from $217 \mathrm{~nm}$ of AL thickness. The FF also improves from the average value of $41.97 \%$ for the devices with the thinnest AL (101 $\mathrm{nm}$ ) to $47.10 \%$ on average for the devices with $116 \mathrm{~nm}$ of $\mathrm{AL}$, then it stays at a similar constant value regardless of the AL thickness increase. This implies that the AL in that thickness range does not contribute to an increase of the average series resistance of the devices demonstrating the capability of PM6:Y6 to keep a high performance even coating with thick active layers. The highest PCE obtained in this thickness optimization section is $6.26 \%$, with an FF of $49.01 \%$, when coating with ink concentration of $24 \mathrm{mg} / \mathrm{ml}$, equivalent to $217 \mathrm{~nm}$ thickness. The average PCE for that $\mathrm{AL}$ thickness is $5.82 \%$ (Table 2), the highest average of this section.
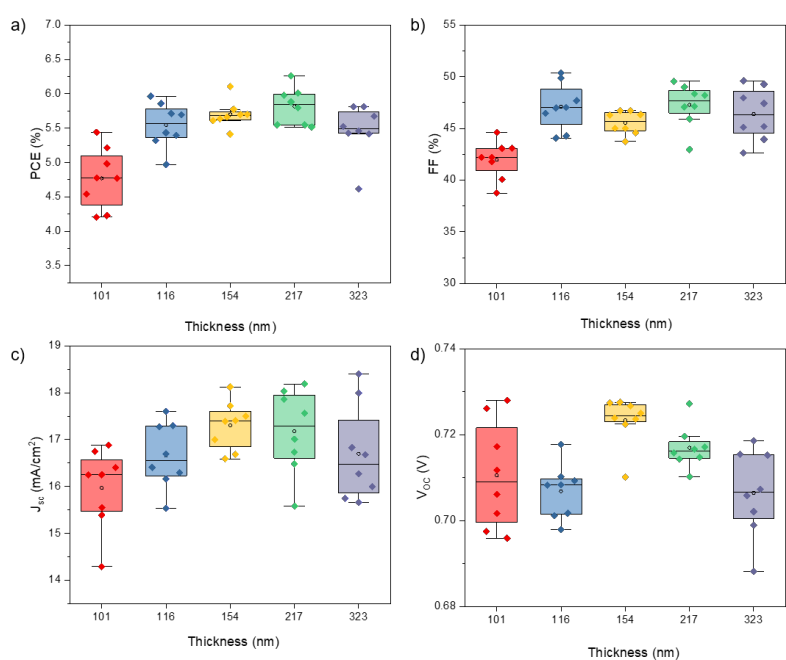

Figure 5: Box plots of a) PCE and b) FF c) Jsc and d) Voc of PM6:Y6 devices with different AL thicknesses.

From the plot $\mathrm{J}_{\mathrm{sc}}$ versus $\mathrm{P}_{\text {in }}$ (Figure $\mathrm{S} 8 \mathrm{a}$ ) we observe that for the solar cells with different $A L$ thicknesses coated at $100 \mathrm{C}$, the solar cell with $217 \mathrm{~nm}$ of AL thickness has the lower charge-transport limited behavior is due to bimolecular recombination with a slope $\mathrm{S}=0.987$, 
a)

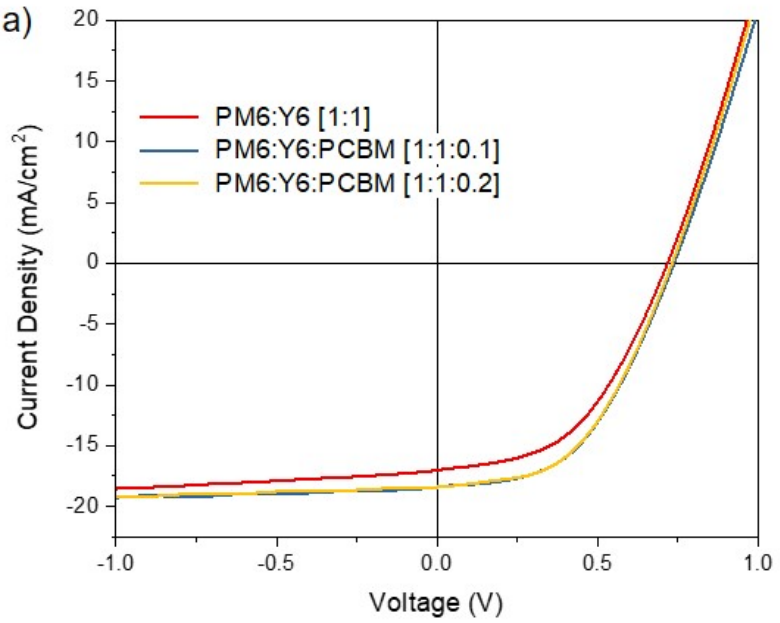

b)

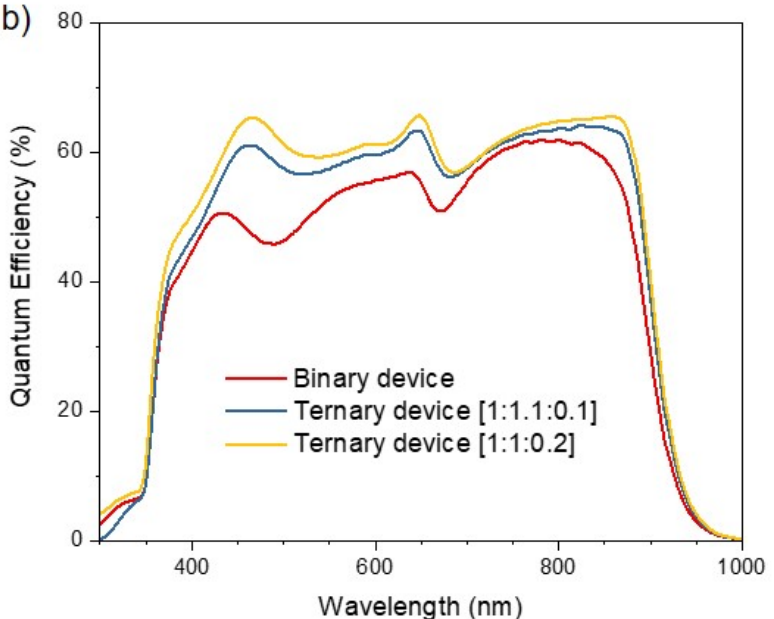

Figure 6: a) J-V curve comparison between binary and ternary devices and b) QE comparison of PM6:Y6 based solar cells

the slope value closest to 1 in this optimization study. However, under open-circuit conditions, our solar cells present a high degree of trap-assisted recombination (Figure $\mathrm{S} 8 \mathrm{~b}$ ). In that type of recombination electrons and holes recombine in trap states or recombination centers within the materials (due to impurities in the materials) or at the interfaces. ${ }^{36}$ Since our devices are prepared not in a cleanroom or inside of a glovebox it is not surprising that this type of recombination is high in open-air processed flexible devices.

\section{Ternary PM6:Y6: $\mathrm{PC}_{61} \mathrm{BM}$ devices}

The efficiency of PM6:Y6 flexible devices can be increased even further by introducing a ternary strategy at the optimal coating temperature and $\mathrm{AL}$ thickness, using a fullerene $\left(\mathrm{PC}_{61} \mathrm{BM}\right)$ as a complementary acceptor. It has been shown that fullerenes enable better electron mobility and more balanced charge transport. ${ }^{37}$ The LUMO level of $\mathrm{PC}_{61} \mathrm{BM}$ is between -3.7 and $-3.9 \mathrm{eV}$, located in between the LUMO levels of PM6 and Y6, generating an energy cascade effect, which is favorable to obtain enhanced values of $J_{S C}$ and $\mathrm{V}_{\mathrm{OC}}{ }^{38}$. Following previous works based on ternary PM6:Y6 blends with fullerenes, ${ }^{39-41}$ we started with a [1:1:0.2] ratio. Moreover, we also reduced the amount of PCBM in the blend by half, to a [1:1.1:0.1] ratio, to determine the amount of $\mathrm{PC}_{61} \mathrm{BM}$ that leads to optimal device performance.

The respective J-V Curves are shown in Figure 6a and their average values are shown in Table $\mathrm{S} 2$. An enhanced $\mathrm{J}_{\mathrm{sc}}$ is clearly visible when a small amount of fullerene acceptor is added to the blend.

Short current circuit values of up to $20 \mathrm{~mA} / \mathrm{cm}^{2}$ are measured on devices with the [1:1:0.2] ternary blend. On average a $12 \%$ higher $\mathrm{J}_{\mathrm{SC}}$ is obtained from the [1:1:0.2] ternary blend compared with binary devices coated at the same temperature $\left(100^{\circ} \mathrm{C}\right)$ with a $24 \mathrm{mg} / \mathrm{mL}$ concentration in o-xylene (Table S2). Both tested ratios performed at a similar level with $\mathrm{PC}_{61} \mathrm{BM}$ as the third component. The average $\mathrm{PCE}$ of the [1:1:0.2] and [1:1.1:0.1] ternary devices are $6.61 \%$ and $6.65 \%$, respectively. The slightly higher average PCE for the [1:1.1:0.1] ternary devices are due to an average higher $\mathrm{V}_{\mathrm{OC}}$ and FF of $0.733 \mathrm{~V}$ and $49.10 \%$, respectively compared to $0.725 \mathrm{~V}$ and $47.31 \%$, respectively for the [1:1:0.2] blends. An only small amount of ternary compound added to the blend has a positive effect on the current and the FF, and therefore an impact on the overall efficiency of the devices.

When $\mathrm{PC}_{61} \mathrm{BM}$ is added to the blend as a complementary acceptor, the EQEs (Figure 6b) do not show any specific photocurrent maximum at a specific wavelength region that could be associated with the photocurrent contribution of PCBM because of the weak however broad absorption of the fullerene acceptor ${ }^{42}$. For both ternary blend formulations tested here, keeping the donor/acceptor ratio constant, we observe a photocurrent gain in all the spectral regions where the organic layer is photoactive. Figure 7 shows a box plot comparing the main photovoltaic parameters of the binary and ternary devices.
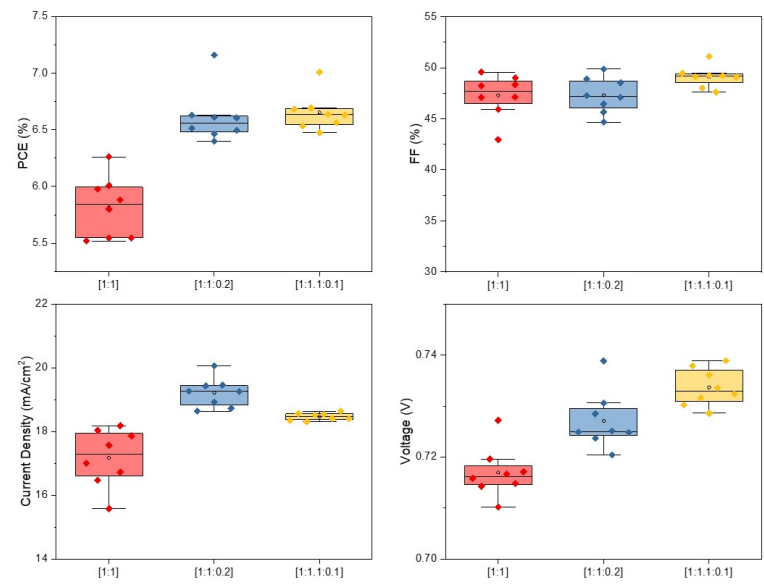

Figure 7. Comparison of PV parameters between binary and ternary devices for a) PCE, b) FF, c) current density $\left(\mathrm{J}_{\mathrm{sc}}\right)$ and $\mathrm{d}$ ) open-circuit voltage $\left(\mathrm{V}_{\mathrm{oc}}\right)$. 

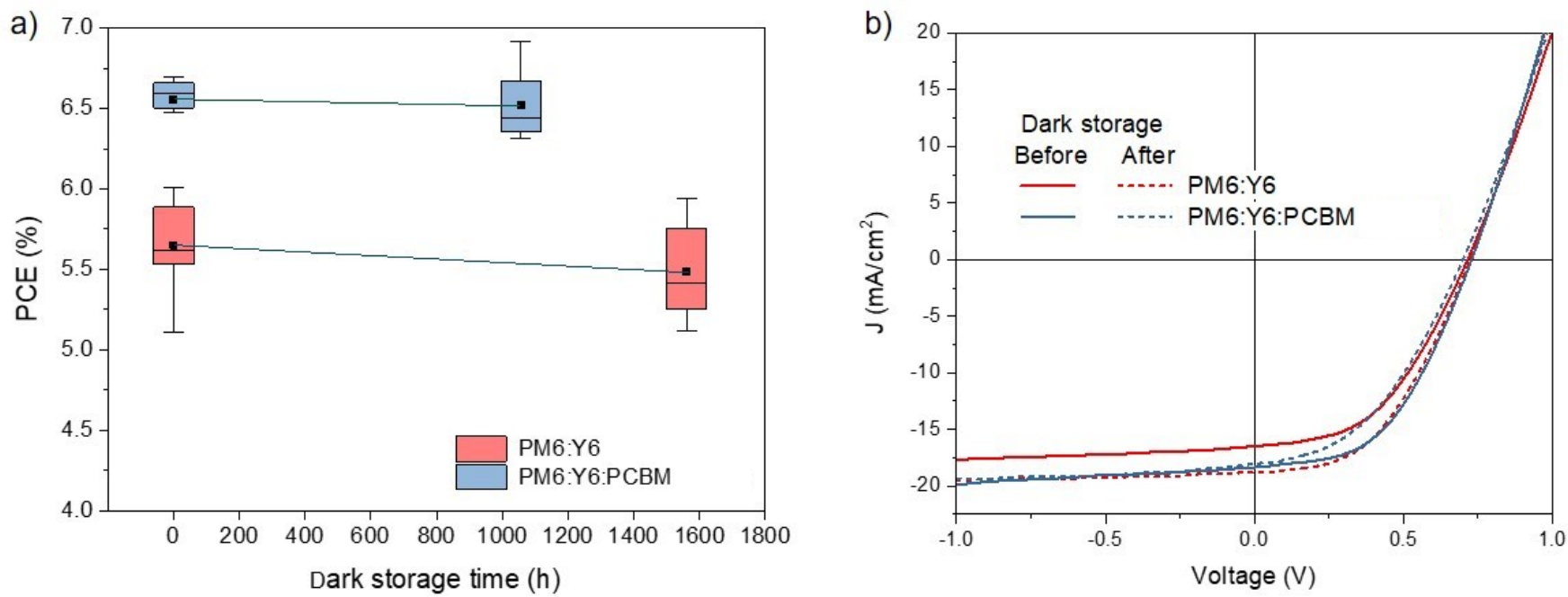

Figure 8: a) Stability of PM6:Y6 devices under dark storage and b) J-V curves of corresponding devices after and before storage

Then, we calculated the exciton dissociation and the charge collection efficiencies $\left(\eta_{\text {diss }}\right.$ and $\left.\eta_{\text {coll }}\right)$ of the binary and ternary devices to reveal the reason for the performance improvement from a photophysical viewpoint. In order to investigate that, the photocurrent density $\left(\mathrm{J}_{\mathrm{ph}}\right)$ versus the effective voltage $\left(\mathrm{V}_{\text {eff }}\right)$ curves are plotted and analyzed. The photocurrent density is defined as the difference between the current density under illumination $\left(\mathrm{J}_{\mathrm{L}}\right)$ and the dark current density $\left(\mathrm{J}_{\mathrm{D}}\right)$. While the effective voltage is defined as the voltage at zero $\mathrm{Jph}_{\mathrm{ph}}$ (or $\mathrm{V}_{\mathrm{OC}}$ ) minus the applied voltage bias $\left(\mathrm{V}_{\text {appl }}\right)$. From the plot presented in Figure S9, it is possible to designate the saturated current density $\left(\mathrm{J}_{\mathrm{sat}}\right)$ from the saturation value for $J_{\mathrm{ph}}$, where the generated excitons are fully collected, normally reached when $V_{\text {eff }}>2 \mathrm{~V}$. We calculate $\eta_{\text {diss }}$ and $\eta_{\text {coll }}$ from $J_{\text {sc }} / J_{\text {sat }}$ and $J_{\text {max }} /$ sat respectively (Table S3) ${ }^{43,44}$. The dissociation probability is $93.7 \%$ for the binary device while it is $96.3 \%$ and $95.7 \%$ for the ternary $1: 1: 0.2$ and 1:1.1:0.1 devices respectively. It shows that one of the benefits of adding PCBM to the blend is the enhancement of the dissociation probability possibly due to the existence of additional and energetically favorable donor/acceptor interfaces. As expected from the higher current density of the ternary devices the collection probability is also improved from $69.6 \%$ for the binary device to $77.7 \%$ and $73.2 \%$ for the ternary $1: 1: 0.2$ and 1:1.1:0.1 devices respectively confirming for the first time that a better charge extraction can be achieved with a small acceptor fullerene amount in OSCs in flexible substrates and fabricated using slot-die coating in a roll-platform.

\section{Stability}

The stability under dark storage for our devices was determined by measuring the encapsulated devices after a certain time stored in dark (up to more than two months), at normal room conditions following the ISOS-D-1 protocol (Figure 8) ${ }^{45}$. The average PCES measured after 1050 hours for the ternary device kept $99.4 \%$ of the average initial value, whereas the average PCEs of binary devices kept more than $97 \%$ of the initial average PCE after more than 1500 hours of storage. It is illustrated in Figure 8 a with the lines connecting the means of the statistical PCE data of pristine and aged devices. It is worth mentioning that some of the devices showed improved performance after storage time in the case of the ternary device.

\section{Conclusions}

Binary and ternary BHJ PM6:Y6-based OSCs with average PCE of $5.82 \%$ (up to $6.26 \%$ ) and $6.65 \%$ (champion efficiency of $7.21 \%$ ) respectively are demonstrated. The fabrication and optimization were done through slot-die coating in a roll-platform in open-air, using green solvents without additives. This demonstration paves the way for upscaling the production of OSCs based on the outstanding PM6:Y6 candidate. In order to optimize our devices, we study the effect of the coating temperature. Due to the temperature aggregation behavior of the blend, we used the so-called hot slot-die coating from hot solution, finding that by matching the flexible substrate temperature with that of the hot slot-die head at $100^{\circ} \mathrm{C}$, the PCE has a maximum due to reduced bimolecular recombination resulting in a higher short-circuit current. The optimal active layer thickness is found to be around $\sim 200 \mathrm{~nm}$. However, the FF remains almost unaffected on devices with active layer thickens from $\sim 100$ to $\sim 300 \mathrm{~nm}$. We confirm that also for the case of PM6:Y6 roll-coated in open-air a small amount of $\mathrm{PC}_{61} \mathrm{BM}$ improves the $\mathrm{J}_{\mathrm{sc}}$ due to a photocurrent increase in all the photoactive region of the blend (360$900 \mathrm{~nm})$. It is found to be related to a $10 \%$ increase in the collection probability in the ternary blends.

In this work, we demonstrate the use of scalable techniques to fabricate high-efficiency OSCs with an active area of $\approx 0.8 \mathrm{~cm}^{2}$ (measured with $\approx 0.4 \mathrm{~cm}^{2}$ shadow mask), to abridge the lab-to-fab gap of the mainstream reports using spin-coated films with active areas $<0.05 \mathrm{~cm}^{2}$ fabricated in a glovebox. The small area devices prepared in controlled environments have taught us many important lessons that allow progress in other areas, and with this work, we aim at awakening the interests of other research groups on the big and important challenges of larger area OSCs. 


\section{Author Contributions}

Marcial Fernández Castro and Jean Truer worked on the experimental fabrication and characterization of the OSCs, analysed data and contributed to the writing of this manuscript.

Moisés Espíndola-Rodríguez supervised the experimental work, helped analysing the data and contributed to the writing of this manuscript.

Jens Wenzel Andreasen supervised this work and helped with the reviewing and editing of the manuscript.

\section{Conflicts of interest}

There are no conflicts to declare.

\section{Acknowledgements}

We gratefully acknowledge funding from the European Research Council (ERC) under the European Union's Horizon 2020 research and innovation programme (SEEWHI - Solar Energy Enabled for the World by High Resolution Imaging, Consolidator Grant No. 681881).

We thank Kristian Larsen for all the technical help that made possible to carry out this scientific publication.

Special acknowledgement to Shinhee Yun (DTU Energy) for conducting the AFM measurements, necessary to have precise thickness measurements in our devices.

\section{References}

1. Guo, Q. et al. Recent advances in PM6:Y6-based organic solar cells. Materials Chemistry Frontiers 5, 3257-3280 (2021).

2. Li, Y. et al. A Simple Phenyl Group Introduced at the Tail of Alkyl Side Chains of Small Molecular Acceptors: New Strategy to Balance the Crystallinity of Acceptors and Miscibility of Bulk Heterojunction Enabling Highly Efficient Organic Solar Cells. Advanced Materials 31, 1-12 (2019).

3. Liu, T. et al. Optimized Fibril Network Morphology by Precise Side-Chain Engineering to Achieve HighPerformance Bulk-Heterojunction Organic Solar Cells. Advanced Materials 30, 1-8 (2018).

4. Pang, Z. et al. Insight into the effects of alkoxy side chain position in nonfullerene electron acceptors on the morphological stability of organic solar cells. Dyes and Pigments 181, 108562 (2020).

5. Armin, A. et al. A History and Perspective of Non-Fullerene Electron Acceptors for Organic Solar Cells. Advanced Energy Materials 11, 1-42 (2021).

6. Gurney, R. S., Lidzey, D. G. \& Wang, T. A review of nonfullerene polymer solar cells: From device physics to morphology control. Reports on Progress in Physics 82, (2019).

7. Lucera, L. et al. Printed semi-transparent large area organic photovoltaic modules with power conversion efficiencies of close to $5 \%$. Organic Electronics 45, 209-214 (2017).

8. Zhang, J. et al. Highly Efficient Semitransparent Organic Solar Cells with Color Rendering Index Approaching 100 Advanced Materials 31, 1-9 (2019).

9. Shi, H., Xia, R., Zhang, G., Yip, H. L. \& Cao, Y. Spectral Engineering of Semitransparent Polymer Solar Cells for Greenhouse Applications. Advanced Energy Materials 9, 18 (2019).

10. Chen, X. et al. Realizing Ultrahigh Mechanical Flexibility and $>15 \%$ Efficiency of Flexible Organic Solar Cells via a "Welding" Flexible Transparent Electrode. Advanced Materials 32, 1-10 (2020).

11. Gao, K. et al. Low-Bandgap Porphyrins for Highly Efficient Organic Solar Cells: Materials, Morphology, and Applications. Advanced Materials 32, 1-19 (2020).

12. Gertsen, A. S., Fernandez Castro, M., Søndergaard, R. R. \& Andreasen, J. W. Scalable fabrication of organic solar cells based on non-fullerene acceptors. Flexible and Printed Electronics 5, 014004 (2019).

13. Yang, F., Huang, Y., Li, Y. \& Li, Y. Large-area flexible organic solar cells. npj Flexible Electronics 5, 1-12 (2021).

14. Xue, P., Cheng, P., Han, R. P. S. \& Zhan, X. Printing fabrication of large-area non-fullerene organic solar cells. Materials Horizons (2021) doi:10.1039/d1mh01317c.

15. Li, Y. et al. Additive-free organic solar cells with enhanced efficiency enabled by unidirectional printing flow of high shear rate. Organic Electronics 97, 106274 (2021).

16. Zhao, H. et al. Processing-Friendly Slot-Die-Cast Nonfullerene Organic Solar Cells with Optimized Morphology. ACS Applied Materials and Interfaces (2019) doi:10.1021/acsami.9b12522.

17. Fernández Castro, M., Mazzolini, E., Sondergaard, R. R., Espindola-Rodriguez, M. \& Andreasen, J. W. Flexible ITOfree roll-processed large-area nonfullerene organic solar cells based on P3HT:O-IDTBR. Physical Review Applied 14, 1 (2020).

18. Zhao, H. et al. Hot Hydrocarbon-Solvent Slot-Die Coating Enables High-Efficiency Organic Solar Cells with Temperature-Dependent Aggregation Behavior. Advanced Materials 32, 2002302 (2020).

19. Würfel, U. et al. A $1 \mathrm{~cm} 2$ Organic Solar Cell with $15.2 \%$ Certified Efficiency: Detailed Characterization and Identification of Optimization Potential. Solar RRL 5, (2021). 
20. Liu, X. et al. Non-Halogenated Polymer Donor-Based Organic Solar Cells with a Nearly 15\% Efficiency Enabled by a Classic Ternary Strategy. ACS Applied Energy Materials 4, 1774-1783 (2021).

21. Na, S.-I. et al. High Performance Roll-to-Roll Produced Fullerene-Free Organic Photovoltaic Devices via Temperature-Controlled Slot Die Coating. Advanced Functional Materials 29, 1805825 (2019).

22. Meng, X. et al. A General Approach for Lab-toManufacturing Translation on Flexible Organic Solar Cells. Advanced Materials 31, 1-10 (2019).

23. Liu, Q. et al. 18\% Efficiency organic solar cells. Science Bulletin 65, 272-275 (2020).

24. Chang, M. et al. Achieving an Efficient and Stable Morphology in Organic Solar Cells Via Fine-Tuning the Side Chains of Small-Molecule Acceptors. Chemistry of Materials 32, 2593-2604 (2020).

25. Li, W. et al. Molecular Order Control of Non-fullerene Acceptors for High-Efficiency Polymer Solar Cells. Joule 3, 819-833 (2019).

26. Wang, W. et al. Controlling Molecular Mass of Low-BandGap Polymer Acceptors for High-Performance All-Polymer Solar Cells. Joule 4, 1070-1086 (2020).

27. Liang, Z. et al. Optimization Requirements of Efficient Polythiophene:Nonfullerene Organic Solar Cells. Joule 4, 1278-1295 (2020).

28. Liao, H. C. et al. Additives for morphology control in highefficiency organic solar cells. Materials Today 16, 326-336 (2013).

29. Xie, Y. et al. Morphology Control Enables Efficient Ternary Organic Solar Cells. Advanced Materials 30, 1-8 (2018).

30. Zhao, F., Wang, C. \& Zhan, X. Morphology Control in Organic Solar Cells. Advanced Energy Materials 8, 1703147 (2018).

31. Song, X. et al. Controlling Blend Morphology for Ultrahigh Current Density in Nonfullerene Acceptor-Based Organic Solar Cells. ACS Energy Letters 3, 669-676 (2018).

32. Cowan, S. R., Roy, A. \& Heeger, A. J. Recombination in polymer-fullerene bulk heterojunction solar cells. Physical Review B - Condensed Matter and Materials Physics 82, 245207 (2010).

33. Hu, Z. et al. Semitransparent organic solar cells exhibiting $13.02 \%$ efficiency and $20.2 \%$ average visible transmittance. Journal of Materials Chemistry A 9, 6797-6804 (2021).

34. Cui, Y. et al. Efficient Semitransparent Organic Solar Cells with Tunable Color enabled by an Ultralow-Bandgap Nonfullerene Acceptor. Advanced Materials 29, 1-7 (2017).
35. Jiang, T. et al. Semitransparent organic solar cells based on all-low-bandgap donor and acceptor materials and their performance potential. Materials Today Energy 21, (2021).

36. Kyaw, A. K. K. et al. Intensity Dependence of CurrentVoltage Characteristics and Recombination in HighEfficiency Solution-Processed Small-Molecule Solar Cells. ACS nano 7, 4569-4577 (2013).

37. Yu, R. et al. Improved Charge Transport and Reduced Nonradiative Energy Loss Enable Over 16\% Efficiency in Ternary Polymer Solar Cells. Advanced Materials 31, (2019).

38. Gasparini, N., Salleo, A., McCulloch, I. \& Baran, D. The role of the third component in ternary organic solar cells. Nature Reviews Materials 4, 229-242 (2019).

39. Pan, M. A. et al. 16.7\%-efficiency ternary blended organic photovoltaic cells with PCBM as the acceptor additive to increase the open-circuit voltage and phase purity. Journal of Materials Chemistry A 7, 20713-20722 (2019).

40. Yang, X. et al. A Highly Conductive Titanium Oxynitride Electron-Selective Contact for Efficient Photovoltaic Devices. Advanced Materials 32, (2020).

41. He, S. et al. Vertical Miscibility of Bulk Heterojunction Films Contributes to High Photovoltaic Performance. Advanced Materials Interfaces 7, 1-10 (2020).

42. Nielsen, C. B., Holliday, S., Chen, H. Y., Cryer, S. J. \& McCulloch, I. Non-Fullerene Electron Acceptors for Use in Organic Solar Cells. Accounts of Chemical Research 48, 2803-2812 (2015)

43. Ma, R. et al. Understanding the Effect of End Group Halogenation in Tuning Miscibility and Morphology of High-Performance Small Molecular Acceptors. Solar RRL 4, 1-8 (2020).

44. Jin, L. et al. Boosting Highly Efficient Hydrocarbon SolventProcessed All-Polymer-Based Organic Solar Cells by Modulating Thin-Film Morphology. ACS Applied Materials and Interfaces 13, 34301-34307 (2021).

45. Reese, M. O. et al. Consensus stability testing protocols for organic photovoltaic materials and devices. Solar Energy Materials and Solar Cells 95, 1253-1267 (2011). 